\title{
Marginal zone B-cells, a gatekeeper of innate immunity
}

\author{
Moncef Zouali ${ }^{1,2 *}$ and Yolande Richard ${ }^{3,4}$ \\ 1 INSERM UMR-S 606, Paris, France \\ 2 Université Paris Diderot, Sorbone Paris Cité, Paris, France \\ 3 INSERMu1016, CNRS UMR 8104, Department of Immunology, Cochin Institute, Paris, France \\ ${ }^{4}$ Université Paris Descartes, Sorbonne Paris Cité, Paris, France
}

\section{Edited by:}

Uday Kishore, Brunel University, UK

Reviewed by:

Uday Kishore, Brunel University, UK Anthony George Tsolaki, Brunel

University, UK

\section{*Correspondence:}

Moncef Zouali, INSERM U606, Centre Viggo Petersen, Hôpital Lariboisière, 2, rue Ambroise Pare, 75475 Paris CEDEX 10, France.

e-mail:moncef.zouali@wanadoo.fr
To maintain the integrity of an organism constantly challenged by pathogens, the immune system is endowed with a variety of cell types. B lymphocytes were initially thought to only play a role in the adaptive branch of immunity. However, a number of converging observations revealed that two B-cell subsets, marginal zone (MZ) and B1 cells, exhibit unique developmental and functional characteristics, and can contribute to innate immune responses. In addition to their capacity to mount a local antibody response against type-2Tcell-independent (TI-2) antigens, MZ B-cells can participate to T-cell-dependent (TD) immune responses through the capture and import of blood-borne antigens to follicular areas of the spleen. Here, we discuss the multiple roles of MZ B-cells in humans, non-human primates, and rodents. We also summarize studies - performed in transgenic mice expressing fully human antibodies on their B-cells and in macaques whose infection with Simian immunodeficiency virus (SIV) represents a suitable model for HIV-1 infection in humans - showing that infectious agents have developed strategies to subvert MZ B-cell functions. In these two experimental models, we observed that two microbial superantigens for B-cells (protein A from Staphylococcus aureus and protein L from Peptostreptococcus magnus) as well as inactivated AT-2 virions of HIV-1 and infectious SIV preferentially deplete innate-like B-cells - MZ B-cells and/or B1 B-cells - with different consequences on TI and TD antibody responses. These data revealed that viruses and bacteria have developed strategies to deplete innate-like B-cells during the acute phase of infection and to impair the antibody response. Unraveling the intimate mechanisms responsible for targeting MZ B-cells in humans will be important for understanding disease pathogenesis and for designing novel vaccine strategies.

Keywords: Staphylococcus aureus, Peptostreptococcus magnus, HIV-1, Simian immunodeficiency virus, cynomolgus macaque, superantigen, protein $A$
To maintain the integrity of an organism constantly challenged by pathogens, the innate immune system is endowed with a variety of cell types. Initially, B lymphocytes were thought to play solely a role in the adaptive branch of immunity, with little impact on innate immunity. With further investigation, however, it became evident that B-cells can exert a number of antibody (Ab)independent functions, capturing, and concentrating antigen for presentation, producing cytokines, influencing T-cell, and dendritic cell (DC) responses, contributing distinct functions during the immune response in vivo, affecting lymphoid tissue structures, and, even, participating in tissue repair (Zouali, 2008). A number of recent observations also indicate that B-cells engage in the innate arm of immune defense (Viau and Zouali, 2005;

Abbreviations: $\mathrm{Ab}$, antibody (dies); Ag, antigen; APC, antigen presenting cell; APRIL, a proliferation-inducing ligand; BAFF, B-cell activating factor of the TNF family; DC, dendritic cell; FO, follicular; HIV, human immunodeficiency virus; iNKT, invariant natural killer T-cell; MGZ, marginal zone; MMM, metallophilic macrophage; MZM, marginal zone macrophage; SAg, superantigen; SpA, Staphylococcus aureus protein A; SIV, Simian immunodeficiency virus; TD, T-cell-dependent, TI, T-cell-independent; TLR, Toll-like receptor.
Kearney, 2008). Central to this function are marginal zone (MZ) B-cells and B1 B-cells, two subsets of B lymphocytes that are classified as "innate B lymphocytes" due to unique developmental and functional characteristics. In rodents, these B-cell subsets exhibit an activated phenotype that allows their rapid proliferation and differentiation into Ab-secreting cells upon stimulation with thymus independent (TI) antigens (Ags). They seem to preferentially secrete antibodies of the IgM and IgG3 isotypes. Phenotypically, innate-like murine B lymphocytes express the cell surface molecule CD9, which distinguishes them from conventional follicular (FO) B-cells (Won and Kearney, 2002). Whereas murine B1 lymphocytes locate essentially in peritoneal and pleural cavities, MZ B lymphocytes are found principally in the MZ of the spleen, where they represent $5 \%$ of total splenic B-cells. The MZ is recognized as a microanatomical site that allows the transit of cells leaving the bloodstream through the erythrocyte-rich red pulp and entering the lymphocyte-rich white pulp. Its specialized organization facilitates filtering of blood-borne pathogens, and initiation of innate and adaptive immune responses. However, the MZ shows prominent structural variation in different mammals - well developed in rodents, intermediately in humans and non-human primates, 
and poorly developed in the canine and feline species (Koike et al., 1996). Whereas the white pulp is specialized in the adaptive response including humoral responses to thymus-dependent (TD) Ags, the MZ is more specialized in the response to bloodborne pathogens (bacteria, viruses) and to TI Ags (Kearney, 2008). This functional distinction between TD and TI responses correlates with the presence of FO and MZ B-cells, two specialized B-cell populations. To be fully efficient in mounting $\mathrm{Ab}$ responses, these B-cells must establish, in a well-controlled spatiotemporal manner, interactions with specialized subsets of macrophages, DCs, and fibroblast-like cells and respond to environmental cytokines.

Here, we focus on the role of the MZ B cell subset in immune defense and discuss how infectious agents can deploy strategies to subvert this important arm of immunity in mice, and in human and non-human-primates.

\section{THE MARGINAL ZONE OF THE SPLEEN, FROM STRUCTURE TO FUNCTION}

Several observations indicate that the human spleen plays an important role in defense against infectious agents. For example, the low $\mathrm{Ab}$ response found in young and aging individuals is associated with immaturity and compromised functions of the spleen MZ, respectively (Timens et al., 1989). Second, individuals with splenic dysfunctions or splenectomy are highly susceptible to infection with Streptococcus pneumoniae, Haemophilus influenzae, Neisseria meningitidis, or other encapsulated bacteria, and this susceptibility correlates with a deficit in circulating MZ B-cells and a lack of $\mathrm{Ab}$ responses against these pathogens. Third, patients with common variable immunodeficiency presenting with recurrent infection of the respiratory tract and chronic lung disease have an extremely low frequency of IgM memory B-cells and do not produce anti-polysaccharide IgM (Carsetti et al., 2004). Fourth, splenic dysfunctions may also occur in patients with sickle-cell anemia known to manifest increased susceptibility to infection (Wong et al., 1992) or inflammatory bowel disease (Di Sabatino et al., 2007), in whom both impaired IgM production by MZ Bcells and reduced phagocytosis of opsonized particles prevent the clearance of encapsulated bacteria. Taken together, these observations indicate that the spleen exerts specific protective functions that cannot be totally replaced by other lymphoid organs during adult life. Yet, a normal blood MZ B-cell compartment is present in young children with congenital asplenia, suggesting that $M Z$ $B$ cell precursors can colonize alternative sites to develop (Weller et al., 2004). Similarly, cells with morphology and phenotype of MZ B-cells have been observed in the subcapsular sinus of lymph nodes, under the dome epithelium of Peyer's patches in the gut and in the crypt epithelium of tonsils (Spencer et al., 1998; Dono et al., 2000). It remains to be established whether these cells play a role in the defense against encapsulated bacteria.

\section{MARGINAL ZONE B-CELLS}

In the mouse, $\mathrm{MZ} \mathrm{B}$-cells differ in many respects from the predominating subset of mature re-circulating FO B-cells. Whereas the latter are small surface $(\mathrm{s}) \operatorname{IgM}^{\text {low }}$ sIgD high $\mathrm{B}$-cells, the former are intermediate-sized $\operatorname{sIgM}^{\text {high }}$ sIgD $^{\text {low }}$ cells located in a distinct area surrounding the $\mathrm{B}$ cell follicles and the periarteriolar lymphoid sheath. MZ B-cells exhibit a low re-circulating potential and are involved in type-2 TI responses to lipopolysaccharide Ags that form the major constituent of cell walls of encapsulated bacteria, such as S. pneumoniae, N. meningitidis, and H. influenzae. Ex vivo, purified MZ B-cells respond generally more vigorously to mitogens like LPS, dextran-conjugated anti-IgM or IgD Abs, or CD40 ligation, than FO B-cells. Phenotypically, they exhibit a preactivated state (pyroninophilic cytoplasm, less condensed nuclear chromatin, complement receptor CD $21^{\text {high }}, \mathrm{CD} 23^{\text {low }}, \mathrm{CD} 35^{\text {high }}$, and higher basal levels of CD80 and CD86). Reduced levels of $\mathrm{CD} 23$, a negative regulator of B-cells, on MZ B-cells may allow them to produce a rapid $\mathrm{Ab}$ response in comparison to $\mathrm{FO} \mathrm{B}$-cells. These remarkable features, together with their unique anatomical location in the spleen account for the fact that MZ B-cells are endowed with powerful immunological functions.

In rodents, splenic $\mathrm{MZ} \mathrm{B}$-cells are a heterogeneous population of cells comprising $\approx 80 \%$ of naive B-cells and a minor fraction (20\%) with signs of Ag-selection (Dammers et al., 2000). Naïve MZ B-cells harbor germline-encoded variable region genes and produce polyreactive Abs that play a crucial role in the first-line of defense against blood-borne infections with encapsulated bacteria. Such polyreactive naturally occurring Abs are essential in the primary immune response against pathogens (Zouali, 2009). Thus, by providing a strategically located buffer of polyreactive B-cell receptors (BCRs) in the spleen, naive $\mathrm{MZ}$ B-cells form an integral part of innate immunity.

\section{THE FUNCTIONS OF MZ B-CELLS}

In addition to the involvement of $\mathrm{MZ}$ B-cells in the initial response to TI Ag, other studies demonstrate that activated MZ B-cells are potent protein $\mathrm{Ag}$ presenters to $\mathrm{CD} 4^{+} \mathrm{T}$-cells, and have the ability to induce Ag-specific T-cell clonal expansion both in vitro and in vivo (Attanavanich and Kearney, 2004). Specifically, MZ B-cells can mount rapid and efficient primary responses to soluble protein $\mathrm{Ag}$ and have an extraordinary ability to promote T-cell proliferation and cytokine production after immunization with protein Ag. Within a few hours of in vivo Ag priming, MZ B-cells are far superior to FO B-cells in the activation of naive $\mathrm{CD} 4^{+} \mathrm{T}$-cells in vitro, and higher levels of B7.2 are expressed on the cell surface of MZ compared with FO B-cells after Ag stimulation.

As discussed above, MZ B-cells comprise an innate subset localized to the marginal sinuses of the spleen. Because they have the capacity to respond to specific foreign $\mathrm{Ag}$ more rapidly than FO B-cells, they provide early immune responses to blood-borne particulate Ags. In addition to being an early source of pathogenspecific T-cell-independent IgM, MZ B-cells serve to bridge the innate and adaptive immune systems. For example, studies of the spirochete Borrelia hermsii showed that MZ B-cells directly associate with blood-borne Borrelia spirochetes and are activated in vivo to produce pathogen-specific IgM within $72 \mathrm{~h}$ of infection (Belperron et al., 2005). When mice were depleted from MZ B-cells to determine their contribution to host defense against B. burgdorferi, the deficient animals showed a reduced B. burgdorferi-specific $\operatorname{IgM}$, elevated pathogen burden, and reduced levels of $B$. burgdorferi-specific IgG that correlated with diminished splenic $\mathrm{CD}^{+}$ T-cell responses (Belperron et al., 2007). These results demonstrate a role for MZ B-cells not only in Borrelia-specific IgM production, but also in promoting the early adaptive $\mathrm{T}$ - and $\mathrm{B}$-cell responses 
to this spirochete. They reveal an important role for this B-cell subset in priming splenic T-cells and the subsequent development of T-cell-dependent IgG responses during infection.

Recent studies suggest that in addition to TLR signaling in DCs, direct TLR-mediated activation of B-cells is also required for eliciting humoral immune responses. Thus, murine B-cells can be stimulated in vitro by TLR4 and TLR9 ligands to proliferate and secrete $\mathrm{Ab}$ (Whitlock and Watson, 1979). In vivo, chimeric mice in which only B-cells are deficient in TLR signaling fail to mount $\mathrm{Ab}$ responses to protein Ags given with adjuvants (Pasare and Medzhitov, 2005). Consistent with these observations, agonists to intracellular and extracellular TLRs activate MZ B-cells in vivo and promote their release from the $\mathrm{MZ}$, thus potentially accelerating the kinetics of the Ag-specific IgM response (Rubtsov et al., 2008). In response to TLR agonists, MZ B-cells proliferate and exhibit a phenotypic maturation process characterized by an increased expression of MHC class II, CD40, and CD86 molecules. According to the TLR agonist used, they also display a different cytokine profile (Bialecki et al., 2009). The fact that MZ B-cells are well equipped in TLRs and that their stimulation leads to cell proliferation, maturation (i.e., cytokine production) and Ab production (Rubtsov et al., 2008) further supports the conclusion that they play a role as innate sensors in MZ B cell-mediated immune responses.

Strikingly, MZ B-cells are able to promote optimal immune responses by interacting with other cell types. For instance, they can transport immune complexes to follicular DCs and deposit them on their surface, or process Ag for direct presentation on MHC class II molecules to naïve CD4 ${ }^{+}$T-cells (Martin and Kearney, 2002; Attanavanich and Kearney, 2004). Further evidence comes from studies of invariant NKT (iNKT) cells, a subset of NKT cells that recognize exogenous and self glyco/lipid Ags presented by the non-classical class I molecule CD1d expressed on Ag presenting cells (APC; Bendelac et al., 2007). Upon primary stimulation, iNKT cells produce large amounts of immunoregulatory cytokines, including IFN- $\gamma$ and IL-4 that lead to downstream activation of DC, NK cells, B-cells, and conventional T-cells (Bendelac et al., 2007). In the mouse, iNKT cells localize in the splenic $\mathrm{B}$-cell area, including the MZ. In the presence of accessory cells (DC), MZ B-cells stimulate the release of both IFN- $\gamma$ and IL- 4 by iNKT cells, strongly suggesting that MZ B-cells have the potential to condition iNKT cell functions in vivo (Bialecki et al., 2009). The observation that $\mathrm{MZ}$ B-cells play a role in iNKT cell activation in vivo, together with the fact that the BCR facilitates the uptake of particulate Ags and their transport to CD1d-containing endocytic compartments, reinforce the view that the MZ B cell/iNKT cell interactions play important roles in the immune response.

It is remarkable that the MZ is a dynamic structure in which cell retention and trafficking require specific cell interactions. Histologically, the murine MZ comprises mucosal addressin cell adhesion molecule 1 (MAdCAM-1 ${ }^{+}$) sinus-lining reticular endothelial cells, marginal metallophilic macrophages (MMM) that stain with Abs to the Monocyte/Macrophage Marker (MOMA-1), and SIGN-R1 ${ }^{+}$(specific intracellular adhesion molecule-grabbing non-integrin receptor 1) MZ macrophages (MZM). Apart from MZ B-cells, MZM represent the other predominant cell type of the MZ. They are distinct from MMM which express the marker
MOMA-1 and are located at the border of the marginal and follicular zones. MZM are interspersed in several layers within the MZ, and express the markers MARCO, a scavenger receptor belonging structurally to the class A receptor family, and ER-TR9 which is identical to the C-type lectin SIGN-R1. Mouse MZM are highly phagocytic and responsible for clearance of blood-borne TI antigens and apoptotic material entering the spleen. Whereas MARCO binds a range of microbial Ags, including Staphylococcus aureus and Escherichia coli, SIGN-R1 is the predominant receptor for uptake of $S$. pneumoniae and polysaccharide dextran by MZMs (Lanoue et al., 2004; Birjandi et al., 2011). Importantly, the dialog between MZ B-cells and MZM is essential to maintain the MZ structure and to allow efficient immune responses (Karlsson et al., 2003). For example, once MZM bind pathogen, they establish direct cell-cell interactions with MZ B-cells that are required for potent responses (Chen et al., 2005), allowing the clearance of Leishmania donovani and Listeria monocytogenes (Odermatt et al., 1991; Aichele et al., 2003). In another model system, MZ B-cells were found to regulate the expression of molecules on macrophages that are important for trapping Ag, which, in turn, is required for Ag capture by the B-cells, thus modulating MZM functions that are important to protect against lethal infection (You et al., 2011). Consistently, the decreased frequency of MZM and MZ B-cells seen in old mice correlates with disruption in the marginal sinus and reduced capacity to bind and clear pathogen-like particles (Birjandi et al., 2011). Thus, MZ B-cells not only provide a link between the innate and adaptive immune responses, but they also function as regulatory cells that control the phenotype and function of other cells involved in the acute innate immune response.

\section{HUMAN MZ B-CELLS}

Studies of the Ig genes expressed by MZ B-cells of normal untreated, non-intentionally immunized rodents revealed that the majority of the splenic cells exhibit little mutations in their rearranged $\mathrm{V}_{\mathrm{H}}$ genes and, thus, represent naive $\mathrm{B}$-cells, and that a minor fraction (20\%) of the MZ B-cell pool express somatically mutated $\mathrm{V}_{\mathrm{H}}$ genes (Dammers et al., 2000). These features contrast with the situation in the human spleen where the majority (>85\%) of splenic MZ B-cells are somatically mutated (DunnWalters et al., 1995; Tierens et al., 1999), suggesting that memory B-cells constitute the majority of spleen MZ B-cells. These diversification characteristics could either reflect a heavy load of somatic hypermutation occurring during clonal expansion in the $\mathrm{MZ}$, or a process of somatic hypermutations acquired in germinal centers (GCs) at distant sites before cell migration to the MZ. However, the microanatomical structure of the human spleen differs from that of rats and mice with respect to $\mathrm{MZ}$ organization and the presence of a so-called perifollicular zone surrounding the MZ (Steiniger et al., 2006). In contrast to rodents, the human spleen lacks a marginal sinus, and the MZ surrounds B-cell follicles, but not the PALS (periarteriolar lymphatic sheath) in the T-cell rich area. Yet, an additional compartment, the perifollicular zone, which surrounds $\mathrm{B}$-cell follicles, makes a transition between the $\mathrm{MZ}$ and the red pulp (Figure 1). This zone has a dynamic composition with strong similarities with the red pulp in terms of terminal sinuses, blood-filled spaces, sheathed capillaries without endothelial lining, 


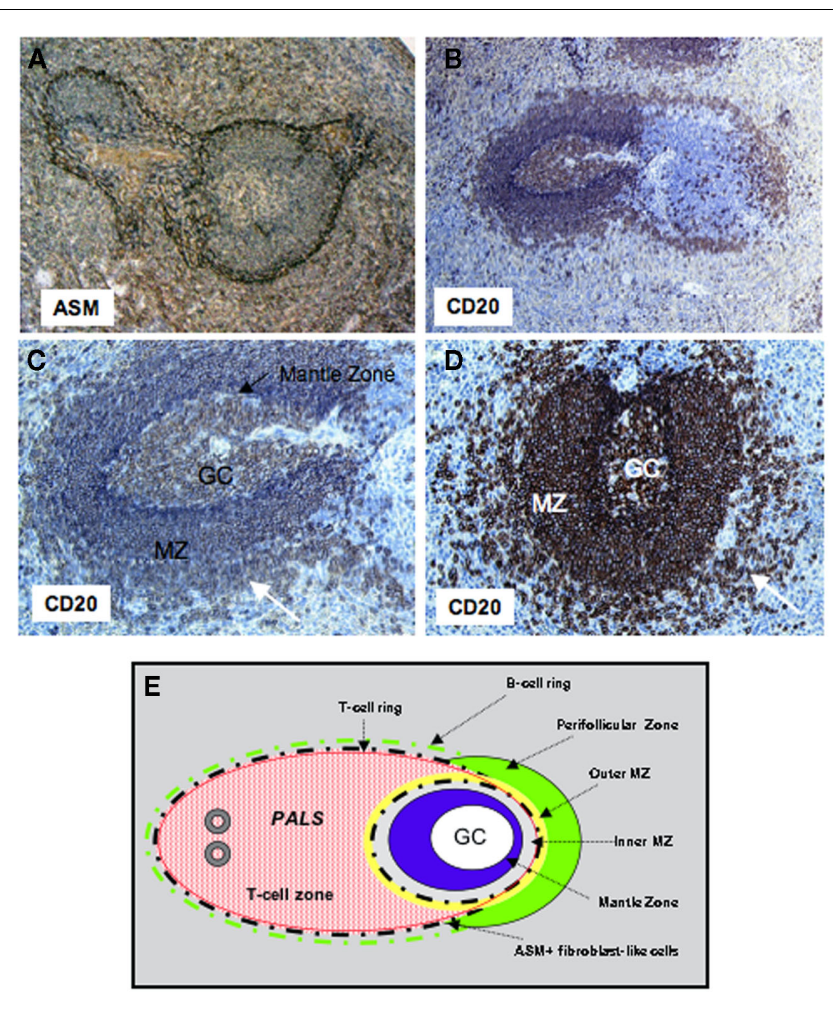

FIGURE 1 | Organization of the follicular and MZ B-cell compartments in human. Sections of human spleens were stained with mAbs to CD20 or alpha smooth muscle actin (ASM). (A) The network of fibroblast-like cells stained by the anti-ASM mAb subdivides the outer and inner MZ around the follicles, and separates the T-cell zone from the red pulp. Original magnification $\times 10$. (B) A ring of B-cells can be seen outside the ASM+ network in both the T and B-cell zones. Original magnification $\times 10$. (C,D) Secondary B-cell follicles with a central germinal center, the mantle zone (intense blue in $\mathrm{C}$, black arrow), the $\mathrm{MZ}$, and the perifollicular zone (white arrow) with less dense accumulation of B-cells. Original magnification $\times 20$. (E) Schematic representation of the T- and B-cell zones in human spleen. GC, germinal center; PALS, periarteriolar lymphatic sheath; MZ, marginal zone.

and scattered B- and T-cells. Because terminal vessels directly open into the perifollicular zone, Ags and lymphocytes likely exit the circulation in this structure and traffic either to the $\mathrm{MZ}$ or to the red pulp (Timens, 1991). A meshwork of fibroblast-like cells, expressing alpha smooth muscle actin (ASM) and MadCAM-1 subdivides the $\mathrm{MZ}$ into large inner and small outer compartments, the latter being in close contact with the perifollicular zone. Along the network of ASM-positive cells, a small ring of B-cells delimits the $\mathrm{T}$-cell zone from the red pulp, whereas a ring of T-cells is frequently present between the inner and outer MZ (Steiniger et al., 2006).

Interestingly, the MZM and MMM subsets found in rodents are lacking in humans. However, macrophages expressing CD68 (lysosome/macrosialin) and CD169 (sialoadhesin) preferentially form sheaths around capillaries in the perifollicular area, but can be also present as scattered cells expressing DC-SIGN (CD209; Steiniger et al., 2006). It remains unclear whether these macrophages can replace $M Z M$ in trapping pathogens and interacting with $M Z$ B-cells. In humans, CD $11 \mathrm{c}^{+} \mathrm{CD} 205^{+}$DCs are intertwined with
MadCAM- $1^{+}$cells at the inner border of the perifollicular zone whereas plasmacytoid DCs are absent from MZ at the steady state (Steiniger et al., 2006; Pack et al., 2008).

Mouse and human MZ B-cells can be distinguished from FO Bcells as being SIgM ${ }^{\text {high }} \mathrm{CD} 21^{\text {high }}$ SIgD $^{\text {low }} \mathrm{CD} 23^{-}$. In humans, MZ $\mathrm{B}$-cells are $\mathrm{CD} 27^{+}$memory B-cells and generally express somatically diversified $\mathrm{V}_{\mathrm{H}}$ genes (Dunn-Walters et al., 1995; Tangye et al., 1998; Weller et al., 2004). Intriguingly, CDR3 spectratyping evidenced a higher clonal diversity in MZ B-cells than in other human memory B-cells (Weller et al., 2008). Starting from less than $1 \%$ in the cord blood, the frequency of MZ B-cells increases concomitantly with that of switched memory blood and spleen B-cells, and the frequency of Ig variable region gene mutation in blood MZ Bcells reaches adult value by $2-4$ years of age. Overall, the available findings suggest that MZ B-cells constitute a pool of pre-diversified $\mathrm{B}$-cells, with a slow rate of renewal, which can be rapidly mobilized by pathogens or polysaccharidic vaccines. Because MZ B-cells persist in CD40 or CD40L-deficient patients, it has been suggested that they develop independently of GCs and T-cells (Weill et al., 2009). The fact that BAFF and APRIL have been shown to support TI responses, activation-induced cytidine deaminase expression and hypermutation, and that BAFF is produced in the MZ may indicate that these cytokines contribute to diversification of $\mathrm{MZ}$ B-cells (Litinskiy et al., 2002).

\section{SUBVERSION OF INNATE-LIKE B CELL IMIMUNITY BY SUPERANTIGENS}

Studies of the confrontation of the mammalian host with infectious agents led to identification of subversion mechanisms that allow pathogens to persist in the organism (Hasler and Zouali, 2003). Such a strategy is used by $S$. aureus to impact MZ B-cells. This bacterium is a leading cause of human infections worldwide. It causes a variety of diseases, including impetigo, cellulitis, food poisoning, toxic shock syndrome, necrotizing pneumonia, endocarditis, and sepsis (Archer, 1998). Our understanding of the molecular mechanisms used by this pathogen to avoid destruction by host defenses remains limited. Even tough $S$. aureus produces a variety of virulence factors, protein $\mathrm{A}(\mathrm{SpA})$ is the prototype staphylococcal surface protein. In addition to its Fc IgG binding capacity, which can be used for Ig purification, SpA also interacts with the $\mathrm{V}_{\mathrm{H}}$ region of Igs, a property responsible for its superantigen (SAg) activity for B-cells (Zouali, 1995, 2007b). To test the effect of SpA in vivo, we used "XMG2L3" transgenic mice whose mouse Ig loci were inactivated and their humoral immune system restored by introduction of human $\mathrm{V}_{\mathrm{H}}, \mathrm{V}_{\mathrm{K}}$, and $\mathrm{V}_{\lambda}$ loci in a germline configuration. As a result, their B lymphocytes express fully human surface Igs, and secrete IgM and IgG2 bearing $\kappa$ or $\lambda$-human L-chains. A remarkable feature of these mice is that half of the $V_{H}$ genes present on the translocus belong to the $V_{H} 3$ gene family, a figure that closely mimics the complexity of the human Ig $\mathrm{V}_{\mathrm{H}}$ locus. When immunized, they mount antigen-specific antibody responses with somatic mutation, isotype switching and affinity maturation.

Analyzing SpA effects in peripheral lymphoid organs, we found that a single injection of SpA to the transgenic humanized mice reduces $\mathrm{B}-1 \mathrm{a}$ cells in the peritoneal cavity and $\mathrm{V}_{\mathrm{H}} 3^{+}$ $\mathrm{B}$-cells in the spleen. Importantly, even repeated administrations 
of this SAg do not modify B-1b or B-2 cell numbers (Viau and Zouali, 2005). In the spleen of treated mice, we studied in detail the fate of various $\mathrm{B}$ cell subpopulations, including newly formed B-cells $\left(\mathrm{B} 220^{+} \mathrm{CD} 23^{-} \mathrm{CD} 21^{-}\right)$, transitional $\mathrm{T} 1\left(\mathrm{~B} 220^{+} \mathrm{CD} 21^{+} \mathrm{IgM}^{+}\right)$, transitional T2 $\left(\mathrm{B} 220^{+} \mathrm{CD} 21^{-} \mathrm{IgM}^{+}\right)$, re-circulating $\mathrm{FO}\left(\mathrm{B} 220^{+} \mathrm{CD} 23^{+} \mathrm{CD} 21^{+}\right)$and resident $\mathrm{MZ} \mathrm{B}-$ cells $\left(\mathrm{B} 220^{+} \mathrm{CD} 23^{-/ \text {low }} \mathrm{CD} 21^{+}\right)$. Remarkably, we found that SpA induced a specific reduction of the $\mathrm{MZ}$ subpopulation (Viau and Zouali, 2005). The molecular basis of this specific deletion of MZ B-cells, as opposed to FO cells, remains unresolved. It is likely that the disparity in responses of $\mathrm{MZ}$ and FO B-cells to SpA reflects a functional dichotomy in these two splenic subpopulations.

In single cell PCR-based studies and sequencing of the expressed genes, we demonstrated that the reduction of $\mathrm{MZ}$ B-cells in SpA-treated mice reflects the decrease of the absolute number of $\mathrm{V}_{\mathrm{H}} 3$-expressing B-cells in this subset (Viau and Zouali, 2005). Thus, the effect is a direct consequence of $\mathrm{V}_{\mathrm{H}} 3^{+} \mathrm{B}$-cell targeting capacity of SpA. To determine whether the SpA B-cell deleting potential had functional consequences, we immunized SpA-treated mice with either a type-2 TI Ag (DNP-Ficoll) or a TD Ag (tetanus toxoid). These studies disclosed a specific impact of SpA on the type-2 TI response (Viau and Zouali, 2005).

It is remarkable that, investigating the in vivo effects of another bacterial SAg, protein L from Peptostreptococcus magnus, we found a similar impact on B-cells with innate-like functions. In contrast to protein A, protein L from P. magnus binds predominantly to $\kappa$-light chains, regardless of the $\mathrm{H}$-chain subclass, and it has affinity for all Ig classes. Since approximately two-thirds of human Igs have $\kappa$-type L-chains, protein L interacts with a significant proportion of Igs. In vitro, protein $\mathrm{L}$ acts as a SAg for human Igs. It also cross-links the $\mathrm{V}_{\mathrm{L}}$ domains of IgE bound to Fce receptors, and stimulates the release of histamine by basophils and mast cells (Zouali, 1995, 2007a). To study protein L effects in vivo, we used "5-feature" transgenic mice whose endogenous loci coding for the $\mathrm{H}$ - and L-chains have been inactivated, and human $\mathrm{H}-, \mathrm{\kappa}$, and $\lambda$ chain transloci were "knocked-in." Humanized mice injected intraperitoneally with $1 \mathrm{mg}$ of recombinant protein $\mathrm{L}$ led to a loss of mature B-cells in the peripheral immune system and a reduction of protein $\mathrm{L}^{+} \mathrm{B}$-cells in the spleen. Further characterization of $\mathrm{B}$-cell subsets in protein L-injected mice revealed a depression of MZ B-cells in the spleen and a reduction of $\mathrm{CD}^{+}$B-cells (B1) in the peritoneal cavity (Viau et al., 2004b).

\section{IMPACT OF HIV-1 ON INNATE-LIKE B-CELLS IN A MODEL OF MICE EXPRESSING FULLY HUMAN IGS}

Although there is no convincing evidence that HIV-1 infects Bcells, marked changes in B-cell responses have been described, including hyper-gammaglobulinemia, increased expression of markers of activation and terminal differentiation, augmented secretion of Igs in vitro and in vivo, and increased susceptibility to apoptosis (Viau and Zouali, 2001; Cagigi et al., 2008). Several longitudinal and cross-sectional studies have indicated that most of these B-cell abnormalities are directly associated with high levels of HIV-1 plasma viremia, and its reduction, by effective anti-retroviral therapy, diminishes polyclonal and HIV-1-specific Ig levels, partially restores APC function and responsiveness to T-cell help. Even efficient anti-retroviral therapy only partially normalizes expression of B-cell activation markers, proportions of memory B-cells, and terminal differentiation (Viau and Zouali, 2001; Cagigi et al., 2008). Thus, B-cell dysfunctions represent a central feature of chronic HIV-1 infection, with potential pathogenic consequences.

Although the mechanisms of these abnormalities remain the focus of much investigation, direct and indirect effects of HIV1 remain possible. One possibility is that the capacity of gp120 to act as SAg for human B-cells could account directly for the biased repertoire expression. In vitro, gp120 binds human $\mathrm{V}_{\mathrm{H}} 3^{+}$ Igs, and activates proliferation and differentiation of $\mathrm{V}_{\mathrm{H}} 3^{+} \mathrm{B}$-cells (Berberian et al., 1993). As it is hard to assess the consequences of these in vitro and ex vivo observations in patients, we used mice into whose genome human Ig loci have been introduced, along with functional inactivation of the endogenous mouse $\operatorname{IgH}$ and Igk loci (Viau et al., 2007). In these transgenic animals, B-cells expressing a virtually full human Ig repertoire develop, allowing investigation of the in vivo consequences of confronting B-cells expressing human Igs with soluble gp120 or inactivated virions. We found that soluble gp120 induced an inversion in the B-1a/B-1b cell ratios, without impacting B-2 cells or affecting substantially the T-cell compartment. Treatment with inactivated AT-2 virions specifically and dramatically depressed B-1a cells, which represent the majority of $\mathrm{B} 1$ cells in normal mice. The observed B-cell changes were associated with a functional alteration of the humoral response to tetanus toxoid. Thus, the results reveal a capacity of HIV-1 to specifically impact a highly specialized B cell subpopulation. Because there is evidence that human IgM memory B-cells are functionally equivalent to murine B-1a cells, our findings suggest that gp120 may have a direct deleting activity on B-cell memory.

\section{IMPACT OF SIMIAN IMMUNODEFICIENCY VIRUS ON MZ B-CELLS IN THE CYNOMOLGUS MACAQUE}

In addition to the presence of immature/transitional $\mathrm{CD} 10^{+}$or exhausted CD27 negative B-cells in the peripheral blood (Ho et al., 2006), alterations in the architecture of GC and splenic $\mathrm{MZ}$ were observed in HIV-infected patients with advanced disease (Legendre et al., 1998; Wilkins et al., 2003). Despite the presence of polyclonal activation, HIV-1-specific Ab responses are strongly impaired, resulting in a decreased response to natural or vaccine TI and TD antigens (Scamurra et al., 2000; Hart et al., 2007), and a loss of peripheral memory B-cells (De Milito et al., 2001; Chong et al., 2004; Titanji et al., 2006). The view that spleen dysfunctions occur during HIV infection is also supported by observations showing that an increased frequency and severity of infection by encapsulated bacteria is a sign of AIDS progression in HIV-1-infected children that has been correlated with histological immaturity or dysfunction of the spleen MZ in HIV-negative children (Timens et al., 1989),

Because recent studies in humans suggest that virus-induced early activation has a major role in shaping the repertoire of memory B-cells and plasma cells, and in trafficking (Badr et al., 2005; Titanji et al., 2005; He et al., 2006; Hart et al., 2007), it became important to examine changes in B-cell subsets in different lymphoid organs during the acute phase of infection. As the study of primary HIV infection in humans is limited by the availability of 

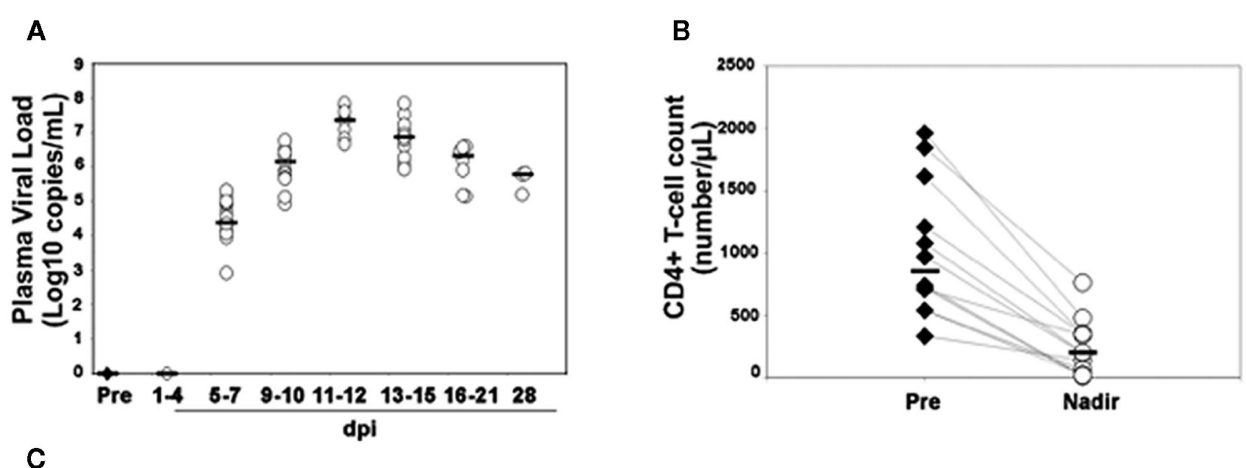

C



FSC

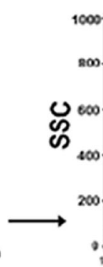

of

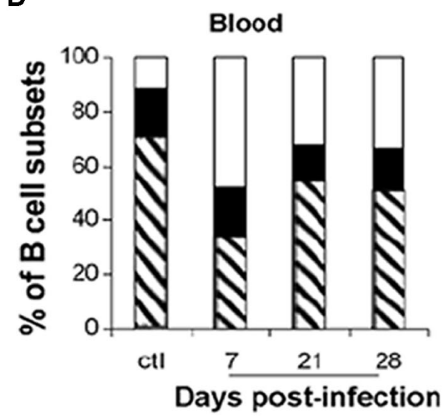

E

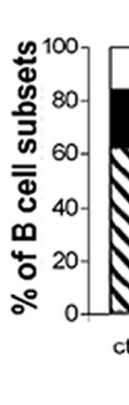

LN

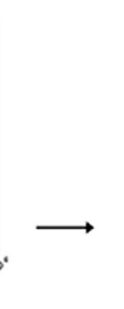

G

CtI

$14 \mathrm{dpi}$

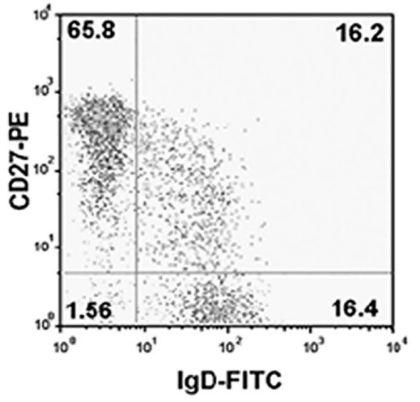

$\mathbf{F}$
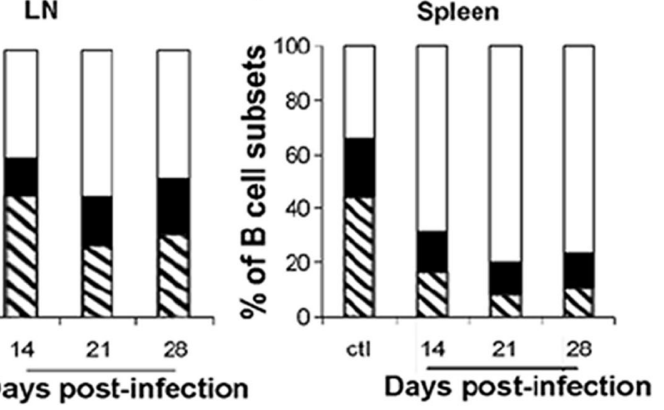

Days post-infection

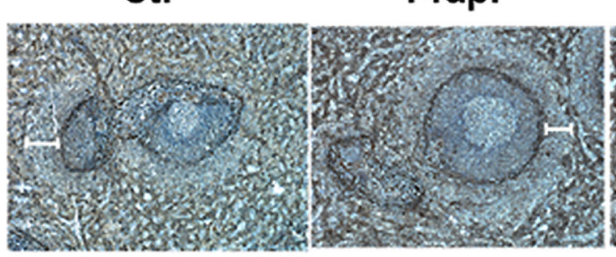

21dpi

28dpi

FIGURE 2 | Loss of MZ B-cells and switched memory B-cells during the acute phase of SIV infection. Macaques were infected intravenously with the pathogenic SIVmac251 strain. For each macaque, blood samples were taken before infection and every 3 days post-infection (dpi). Three groups of five macaques were sacrificed 14, 21, and $28 \mathrm{dpi}$. At euthanasia, spleen, peripheral blood, and mesenteric lymph nodes (LN) were collected from SIV-Infected animals and from four non-infected control animals. (A) Plasma viral load was determined by real-time PCR as described (Peruchon et al., 2009). Horizontal bars indicate median values. (B) Absolute numbers of blood CD4 T-cells were determined in each animal as described (Peruchon et al., 2009) before infection and 11-12 dpi when they reached their lowest values (nadir). Horizontal bars indicate median values. (C) CD27 and SIgD expression was studied by three-parameter immunofluorescence analysis on CD20-gated populations from various organs. Cells were gated on forward and side scatter (R1), and then on CD20 (R2) expression. CD27 and SlgD expression on $\mathrm{CD}_{20} \mathrm{O}^{+}$cells is shown on a representative dot plot of peripheral blood mononuclear cells staining. The percentage of positive cells is indicated in each quadrant. Flow cytometry plots depict $\log _{10}$ fluorescence. (D-F) Shown are the mean percentages of naïve (SlgD+CD27- ${ }^{+}$open area), switched memory (SlgD- ${ }^{-} D 27^{+}$, hatched area), and $\mathrm{MZ}\left(\mathrm{SlgD}{ }^{+} \mathrm{CD} 27^{+}\right.$, black area) B-cells for each group of animals $(n=3)$. (D) Shows data in peripheral blood before and after exposure to SIV. (E) Shows data from the spleen of non-infected animals (ctl, $n=4$ ) and SIV-infected animals ( $n=3$ for all groups). (F) shows data from $L N$ of non-infected animals (ctl, $n=4$ ) and SIV-infected animals ( $n=5$ for all groups). (G) Spleen sections from non-infected macaques (CtI) and from SIV-infected animals collected 14, 21, and 28 dpi were stained with alpha smooth muscle actin (ASM) mAb for visualization of the MZ. Shown are staining for one representative animal from each group. White bars indicate the MZ region width as measured in 19-22 ASM-stained regions in each spleen. Mean values \pm SEM are $132 \pm 14 \mu \mathrm{m}$ for $\mathrm{Ctl}$, and $114 \pm 23,136 \pm 27$ $106 \pm 17 \mu \mathrm{m}$ for macaques infected for 14,21 , and $28 \mathrm{dpi}$, respectively. 
lymphoid organs and by the difficulties associated with performing longitudinal investigations using tissues other than peripheral blood, we used a model of experimental pathogenic infection in cynomolgus macaques by Simian immunodeficiency virus (SIV), a suitable model that reproduces the long-lasting HIV-1 disease (Peruchon et al., 2009). We found a decrease in switched memory and MZ B-cells that differed in intensity and kinetics in the various organs (Figure 2). This decrease correlates with rapid homing of peripheral B-cells into secondary lymphoid organs and their preferential retention in the spleen and the small intestine where SIV replication is particularly intense. Although the histological size of the MZ did not significantly vary, the frequency of MZ B-cells decreased steadily in the spleen and transiently before $21 \mathrm{dpi}$ in peripheral and mesenteric lymph nodes. The MZ B-cell loss was concomitant with a transient peak of apoptosis on $14 \mathrm{dpi}$ and their increased commitment to TI terminal differentiation (Figure 3).

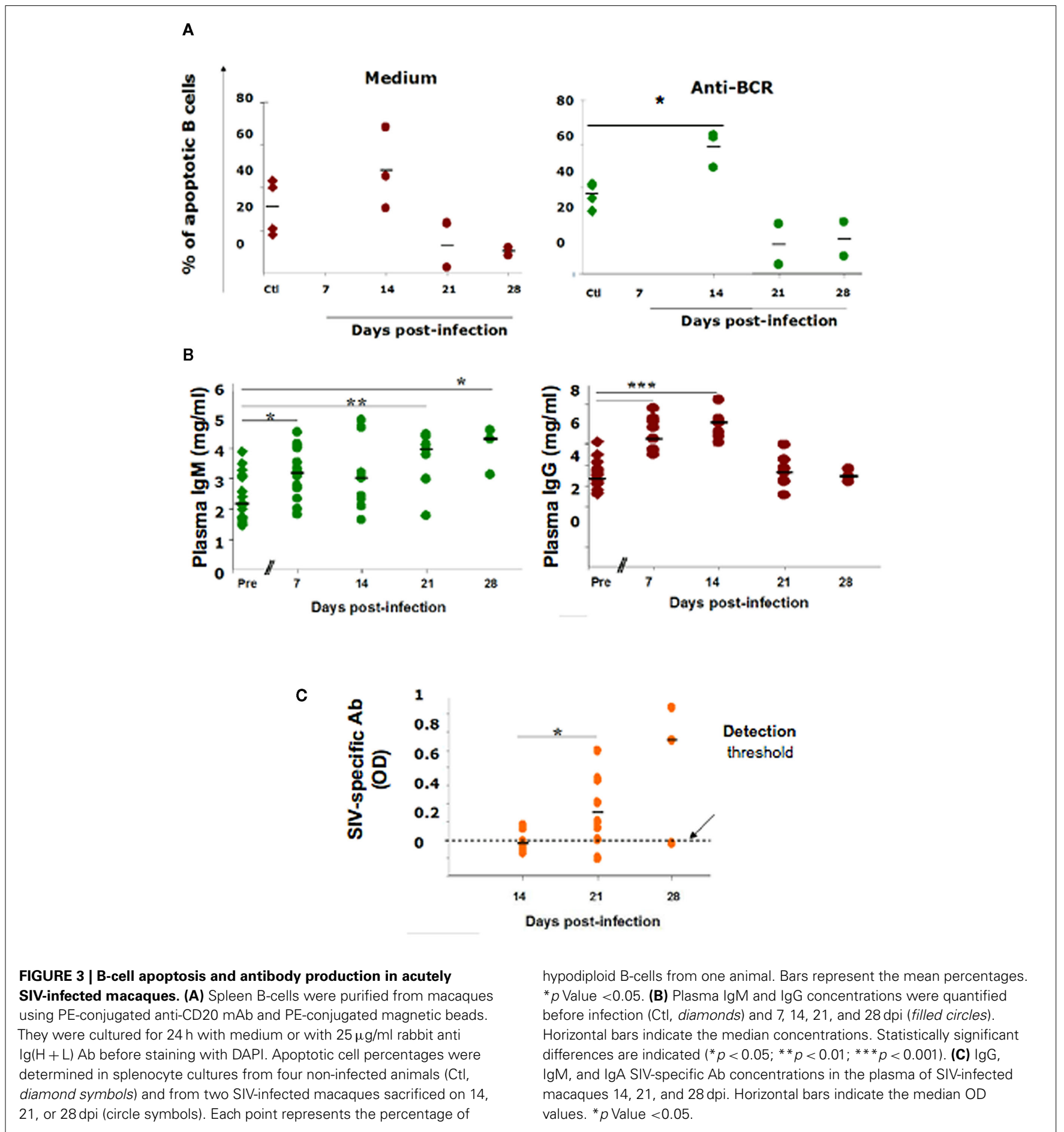


Indeed, we observed an increased density of IgG plasma cells in the $\mathrm{MZ}$ and the red pulp, and augmented plasma IgG and IgM levels before $14 \mathrm{dpi}$. In contrast, new IgG and IgM plasma cells in GCs and plasma SIV-specific Ab were only detectable after 21 dpi. It is possible that SIV-induced soluble FasL and TRAIL, or a direct interaction between gp120 and $\mathrm{V}_{\mathrm{H}} 3$-expressing BCRs contributed to this MZ B-cell apoptosis (Berberian et al., 1993; Karray and Zouali, 1997; Samuelsson et al., 1997; Herbeuval et al., 2006). Since gp120-induced production of BAFF by monocytes can enhance polyclonal IgG production by a subset of MZ Bcells (He et al., 2006), the Ig production we saw would rather be promoted by increased cytokine production, including BAFF and APRIL (Ettinger et al., 2007). Consistently, we observed a transient increase in plasma BAFF levels with a peak on 11-12 dpi. During the acute phase of infection, increased plasma levels correlated with plasma viral load, CD4 T-cell depletion and plasma IgG. In these SIV-infected macaques, no change in plasma APRIL was observed (Yolande Richard, unpublished data). As previous studies in the mouse suggest (Lesley et al., 2004; Thien et al., 2004), BAFF overexpression may also contribute to the preferential production of polyclonal or autoreactive $\mathrm{Ab}$ at the expense of SIV-specific Ab.

As $\mathrm{MZ}$ and switched memory B-cells home together in the spleen MZ, their concomitant depletion during the acute phase of infection is intriguing, and may suggest the existence of a common mechanism. In viremic HIV-infected patients, a sustained BAFF overexpression was postulated to impair the trafficking of B-cells between the MZ and B-cell follicles (Fontaine et al., 2011). In support to this conclusion, we had previously shown that in vitro short-term exposure of human B-cells to BAFF preferentially increased the chemotactic response of $\mathrm{CD} 27^{+} \mathrm{B}$-cells to CXCL13 (Badr et al., 2008). It is possible that type I IFN and BAFF, produced early during the acute phase of infection, may increase the retention of MZ B-cells into B-cell follicles, decrease the import of virus particles, and delay the onset of virus-specific $\mathrm{TD}$ responses. This hypothesis is consistent with SIV-specific Ab

\section{REFERENCES}

Aichele, P., Zinke, J., Grode, L., Schwendener, R. A., Kaufmann, S. H., and Seiler, P. (2003). Macrophages of the splenic marginal zone are essential for trapping of bloodborne particulate antigen but dispensable for induction of specific $\mathrm{T}$ cell responses. J. Immunol. 171, 1148-1155.

Archer, G. L. (1998). Staphylococcus aureus: a well-armed pathogen. Clin. Infect. Dis. 26, 1179-1181.

Attanavanich, K., and Kearney, J. F. (2004). Marginal zone, but not follicular B cells, are potent activators of naive CD4 T cells. J. Immunol. 172, 803-811.

Badr, G., Borhis, G., Lefevre, E. A., Chaoul, N., Deshayes, F., Dessirier, V., Lapree, G., Tsapis, A., and Richard, Y. (2008). BAFF enhances chemotaxis of primary human B cells: a particular synergy between BAFF and CXCL13 on memory B cells. Blood 111, 2744-2754.
Badr, G., Borhis, G., Treton, D., Moog, C., Garraud, O., and Richard, Y. (2005). HIV type 1 glycoprotein 120 inhibits human B cell chemotaxis to $\mathrm{CXC}$ chemokine ligand (CXCL) 12, CC chemokine ligand (CCL)20, and CCL21. J. Immunol. 175, 302-310.

Belperron, A. A., Dailey, C. M., and Bockenstedt, L. K. (2005). Infection-induced marginal zone B cell production of Borrelia hermsiispecific antibody is impaired in the absence of CD1d. J. Immunol. 174, 5681-5686.

Belperron, A. A., Dailey, C. M., Booth, C. J., and Bockenstedt, L. K. (2007). Marginal zone B-cell depletion impairs murine host defense against Borrelia burgdorferi infection. Infect. Immun. 75, 3354-3360.

Bendelac, A., Savage, P. B., and Teyton, L. (2007). The biology of NKT cells. Annu. Rev. Immunol. 25, 297-336.

Berberian, L., Goodglick, L., Kipps, T. J., and Braun, J. (1993).

being detectable in the serum only after the decrease of plasma type I IFN and BAFF (e.g., after $16 \mathrm{dpi}$ ). A better understanding of the mechanisms and consequences of this death on the $\mathrm{Ab}$ response will be essential for vaccine development.

\section{CONCLUSIONS}

As discussed above, MZ B-cells are a first-line of defense against blood-borne pathogens entering the spleen freely through the blood or transported by circulating APCs. They represent the main $\mathrm{B}$-cell population involved in the generation of rapid TI responses to blood-borne bacteria. They play an important role in the production of early $\mathrm{Ab}$ responses against infectious bacterial and virus particles. Splenectomized mice or mice lacking functional MZ Bcells produce delayed $\mathrm{Ab}$ responses to infectious agents that require T-cell collaboration. The easy accessibility of MZ B-cells to bloodborne Ags, their lower threshold of activation, and the enrichment in clones specific for TI antigens account for the critical role that this B-cell subset plays in the induction of rapid $\mathrm{TI} A \mathrm{~b}$ responses.

Taking into account that infectious agents are complex and composed of both TD and TI epitopes, MZ B-cells are likely to be heterogeneous, multireactive, and react with a wide variety of pathogen-associated Ags. Yet, the observations summarized above and obtained in a model of mice expressing human Igs (Viau et al., 2004a,b, 2005, 2007; Viau and Zouali, 2005) and in a non-human primate (Peruchon et al., 2009) revealed that viruses and bacteria have developed strategies to deplete MZ B-cells during the acute phase of infection. Unraveling the intimate mechanisms responsible for targeting MZ B-cells in humans and non-human primates will be important for understanding disease pathogenesis and for designing novel vaccine formulations.

\section{ACKNOWLEDGMENTS}

This work was supported by INSERM (Paris, France) institutional grants and by a grant from the Agence Nationale de Recherches sur le Sida (ANRS, Paris).

Immunoglobulin $\mathrm{VH} 3$ gene products: natural ligands for HIV gp120. Science 261, 1588-1591.

Bialecki, E., Paget, C., Fontaine, J., Capron, M., Trottein, F., and Faveeuw, C. (2009). Role of marginal zone B lymphocytes in invariant NKT cell activation. J. Immunol. 182, 6105-6113.

Birjandi, S. Z., Ippolito, J. A., Ramadorai, A. K., and Witte, P. L. (2011). Alterations in marginal zone macrophages and marginal zone B cells in old mice. J. Immunol. 186, 3441-3451.

Cagigi, A., Nilsson, A., De Milito, A., and Chiodi, F. (2008). B cell immunopathology during HIV1 infection: lessons to learn for HIV-1 vaccine design. Vaccine 26, 3016-3025.

Carsetti, R., Rosado, M. M., and Wardmann, H. (2004). Peripheral development of B cells in mouse and man. Immunol. Rev. 197, 179-191.
Chen, Y., Pikkarainen, T., Elomaa, O., Soininen, R., Kodama, T., Kraal, G. and Tryggvason, K. (2005). Defective microarchitecture of the spleen marginal zone and impaired response to a thymus-independent type 2 antigen in mice lacking scavenger receptors MARCO and SR-A. J. Immunol. 175, 8173-8180.

Chong, Y., Ikematsu, H., Kikuchi, K., Yamamoto, M., Murata, M., Nishimura, M., Nabeshima, S., Kashiwagi, S., and Hayashi, J. (2004). Selective CD27+ (memory) B cell reduction and characteristic B cell alteration in drug-naive and HAART-treated HIV type 1-infected patients. AIDS Res. Hum. Retroviruses 20, 219-226.

Dammers, P. M., Visser, A., Popa, E. R., Nieuwenhuis, P., and Kroese, F. G. (2000). Most marginal zone B cells in rat express germline encoded $\mathrm{Ig}$ $\mathrm{VH}$ genes and are ligand selected. $J$. Immunol. 165, 6156-6169. 
De Milito, A., Morch, C., Sonnerborg, A., and Chiodi, F. (2001). Loss of memory (CD27) B lymphocytes in HIV-1 infection. AIDS 15, 957-964.

Di Sabatino, A., Rosado, M. M., Miele, L., Capolunghi, F., Cazzola, P., Biancheri, P., Carsetti, R., Gasbarrini, G., and Corazza, G. R. (2007). Impairment of splenic IgM-memory but not switched-memory B cells in a patient with celiac disease and splenic atrophy. J. Allergy Clin. Immunol. 120, 1461-1463.

Dono, M., Zupo, S., Leanza, N., Melioli, G., Fogli, M., Melagrana, A., Chiorazzi, N., and Ferrarini, M. (2000). Heterogeneity of tonsillar subepithelial B lymphocytes, the splenic marginal zone equivalents. J. Immunol. 164, 5596-5604.

Dunn-Walters, D. K., Isaacson, P. G., and Spencer, J. (1995). Analysis of mutations in immunoglobulin heavy chain variable region genes of microdissected marginal zone (MGZ) B cells suggests that the MGZ of human spleen is a reservoir of memory B cells. J. Exp. Med. 182, 559-566.

Ettinger, R., Sims, G. P., Robbins, R., Withers, D., Fischer, R. T., Grammer, A. C., Kuchen, S., and Lipsky, P. E. (2007). IL-21 and BAFF/BLyS synergize in stimulating plasma cell differentiation from a unique population of human splenic memory B cells. J. Immunol. 178, 2872-2882.

Fontaine, J., Chagnon-Choquet, J., Valcke, H. S., Poudrier, J., and Roger, M. (2011). High expression levels of B lymphocyte stimulator (BLyS) by dendritic cells correlate with HIVrelated B cell disease progression in humans. Blood 117, 145-155.

Hart, M., Steel, A., Clark, S. A., Moyle, G., Nelson, M., Henderson, D. C., Wilson, R., Gotch, F., Gazzard, B., and Kelleher, P. (2007). Loss of discrete memory B cell subsets is associated with impaired immunization responses in HIV-1 infection and may be a risk factor for invasive pneumococcal disease. J. Immunol. 178, 8212-8220.

Hasler, P., and Zouali, M. (2003). Subversion of B lymphocyte signaling by infectious agents. Genes Immun. 4, 95-103.

He, B., Qiao, X., Klasse, P. J., Chiu, A., Chadburn, A., Knowles, D. M., Moore, J. P., and Cerutti, A. (2006). HIV-1 envelope triggers polyclonal Ig class switch recombination through a CD40independent mechanism involving BAFF and C-type lectin receptors. J. Immunol. 176, 3931-3941.
Herbeuval, J. P., Nilsson, J., Boasso, A., Hardy, A. W., Kruhlak, M. J., Anderson, S. A., Dolan, M. J., Dy, M., Andersson, J., and Shearer, G. M. (2006). Differential expression of IFN-alpha and TRAIL/DR5 in lymphoid tissue of progressor versus nonprogressor HIV-1-infected patients. Proc. Natl. Acad. Sci. U.S.A. 103, 7000-7005.

Ho, J., Moir, S., Malaspina, A., Howell, M. L., Wang, W., Dipoto, A. C., O'Shea, M. A., Roby, G. A., Kwan, R., Mican, J. M., Chun, T. W., and Fauci, A. S. (2006). Two overrepresented B cell populations in HIV-infected individuals undergo apoptosis by different mechanisms. Proc. Natl. Acad. Sci. U.S.A. 103, 19436-19441.

Karlsson, M. C., Guinamard, R., Bolland, S., Sankala, M., Steinman, R. M., and Ravetch, J. V. (2003). Macrophages control the retention and trafficking of B lymphocytes in the splenic marginal zone. J. Exp. Med. 198, 333-340.

Karray, S., and Zouali, M. (1997). Identification of the B cell superantigenbinding site of HIV-1 gp120. Proc. Natl. Acad. Sci. U.S.A. 94, 1356-1360.

Kearney, J. F. (2008). B cell subpopulations and secondary lymphoid organ architecture. Semin. Immunol. 20, $1-3$.

Koike, R., Nishimura, T., Yasumizu, R., Tanaka, H., Hataba, Y., Hataba, Y., Watanabe, T., Miyawaki, S., and Miyasaka, M. (1996). The splenic marginal zone is absent in alymphoplastic aly mutant mice. Eur. J. Immunol. 26, 669-675.

Lanoue, A., Clatworthy, M. R., Smith, P., Green, S., Townsend, M. J., Jolin, H. E., Smith, K. G., Fallon, P. G., and Mckenzie, A. N. (2004). SIGN$\mathrm{R} 1$ contributes to protection against lethal pneumococcal infection in mice. J. Exp. Med. 200, 1383-1393.

Legendre, C., Raphael, M., Gras, G., Lefevre, E. A., Feuillard, J., Dormont, D., and Richard, Y. (1998). CD80 expression is decreased in hyperplastic lymph nodes of HIV+ patients. Int. Immunol. 10, 1847-1851.

Lesley, R., Xu, Y., Kalled, S. L., Hess, D. M., Schwab, S. R., Shu, H. B., and Cyster, J. G. (2004). Reduced competitiveness of autoantigen-engaged $B$ cells due to increased dependence on BAFF. Immunity 20, 441-453.

Litinskiy, M. B., Nardelli, B., Hilbert, D. M., He, B., Schaffer, A., Casali, P., and Cerutti, A. (2002). DCs induce CD40-independent immunoglobulin class switching through BLyS and APRIL. Nat. Immunol. 3, 822-829.
Martin, F., and Kearney, J. F. (2002). Marginal-zone B cells. Nat. Rev. Immunol. 2, 323-335.

Odermatt, B., Eppler, M., Leist, T. P. Hengartner, H., and Zinkernagel, R. M. (1991). Virus-triggered acquired immunodeficiency by cytotoxic T-cell-dependent destruction of antigen-presenting cells and lymph follicle structure. Proc. Natl. Acad. Sci. U.S.A. 88, 8252-8256.

Pack, M., Trumpfheller, C., Thomas, D., Park, C. G., Granelli-Piperno, A., Munz, C., and Steinman, R. M. (2008). DEC-205/CD205+ dendritic cells are abundant in the white pulp of the human spleen, including the border region between the red and white pulp. Immunology 123 438-446.

Pasare, C., and Medzhitov, R. (2005). Control of B-cell responses by Toll-like receptors. Nature 438 , 364-368.

Peruchon, S., Chaoul, N., Burelout, C., Delache, B., Brochard, P., Laurent, P., Cognasse, F., Prevot, S., Garraud, O., Le Grand, R., and Richard, Y. (2009). Tissue-specific B-cell dysfunction and generalized memory B-cell loss during acute SIV infection. PLoS ONE 4, e5966. doi:10.1371/journal.pone.0005966

Rubtsov, A. V., Swanson, C. L., Troy, S., Strauch, P., Pelanda, R., and Torres, R. M. (2008). TLR agonists promote marginal zone $\mathrm{B}$ cell activation and facilitate $\mathrm{T}$-dependent IgM responses. J. Immunol. 180, 3882-3888.

Samuelsson, A., Sonnerborg, A., Heuts, N., Coster, J., and Chiodi, F. (1997). Progressive B cell apoptosis and expression of Fas ligand during human immunodeficiency virus type 1 infection. AIDS Res. Hum. Retroviruses 13, 1031-1038.

Scamurra, R. W., Miller, D. J., Dahl, L., Abrahamsen, M., Kapur, V., Wahl, S. M., Milner, E. C., and Janoff, E. N. (2000). Impact of HIV-1 infection on $\mathrm{VH} 3$ gene repertoire of naive human B cells. J. Immunol. 164, 5482-5491.

Spencer, J., Perry, M. E., and DunnWalters, D. K. (1998). Human marginal-zone B cells. Immunol. Today 19, 421-426.

Steiniger, B., Timphus, E. M., and Barth, P. J. (2006). The splenic marginal zone in humans and rodents: an enigmatic compartment and its inhabitants. Histochem. Cell Biol. 126, 641-648.

Tangye, S. G., Liu, Y. J., Aversa, G., Phillips, J. H., and De Vries, J. E. (1998). Identification of functional human splenic memory B cells by expression of CD148 and CD27. J. Exp. Med. 188, 1691-1703.

Thien, M., Phan, T. G., Gardam, S., Amesbury, M., Basten, A., Mackay, F., and Brink, R. (2004). Excess BAFF rescues self-reactive $B$ cells from peripheral deletion and allows them to enter forbidden follicular and marginal zone niches. Immunity 20, 785-798.

Tierens, A., Delabie, J., Michiels, L., Vandenberghe, P., and De Wolf-Peeters C. (1999). Marginal-zone B cells in the human lymph node and spleen show somatic hypermutations and display clonal expansion. Blood 93, 226-234.

Timens, W. (1991). The human spleen and the immune system: not just another lymphoid organ. Res. Immunol. 142, 316-320.

Timens, W., Boes, A., RozeboomUiterwijk, T., and Poppema, S. (1989). Immaturity of the human splenic marginal zone in infancy. Possible contribution to the deficient infant immune response. $J$. Immunol. 143, 3200-3206.

Titanji, K., Chiodi, F., Bellocco, R., Schepis, D., Osorio, L., Tassandin, C., Tambussi, G., Grutzmeier, S., Lopalco, L., and De Milito, A. (2005). Primary HIV-1 infection sets the stage for important $B$ lymphocyte dysfunctions. AIDS 19, 1947-1955.

Titanji, K., De Milito, A., Cagigi, A., Thorstensson, R., Grutzmeier, S., Atlas, A., Hejdeman, B., Kroon, F. P., Lopalco, L., Nilsson, A., and Chiodi, F. (2006). Loss of memory $B$ cells impairs maintenance of longterm serologic memory during HIV1 infection. Blood 108, 1580-1587.

Viau, M., Cholley, B., Bjorck, L., and Zouali, M. (2004a). Downmodulation of the antigen receptor by a superantigen for human B cells. Immunol. Lett. 92, 91-96.

Viau, M., Longo, N. S., Lipsky, P. E., Bjorck, L., and Zouali, M. (2004b). Specific in vivo deletion of B-cell subpopulations expressing human immunoglobulins by the Bcell superantigen protein L. Infect. Immun. 72, 3515-3523.

Viau, M., Longo, N. S., Lipsky, P. E., and Zouali, M. (2005). Staphylococcal protein a deletes B-1a and marginal zone B lymphocytes expressing human immunoglobulins: an immune evasion mechanism. J. Immunol. 175, 7719-7727.

Viau, M., Veas, F., and Zouali, M. (2007). Direct impact of inactivated HIV-1 virions on B lymphocyte subsets. Mol. Immunol. 44, 2134-2144. 
Viau, M., and Zouali, M. (2001). Molecular determinants of the human antibody response to HIV-1: implications for disease control. J. Clin. Immunol. 21, 410-419.

Viau, M., and Zouali, M. (2005). Blymphocytes, innate immunity, and autoimmunity. Clin. Immunol. 114, 17-26.

Weill, J., Weller, S., and Reynaud, C. (2009). Human marginal zone B cells. Annu. Rev. Immunol. 27, 267-285.

Weller, S., Braun, M. C., Tan, B. K., Rosenwald, A., Cordier, C., Conley, M. E., Plebani, A., Kumararatne, D. S., Bonnet, D., Tournilhac, O., Tchernia, G., Steiniger, B., Staudt, L. M., Casanova, J. L., Reynaud, C. A., and Weill, J. C. (2004). Human blood IgM "memory" B cells are circulating splenic marginal zone $\mathrm{B}$ cells harboring a prediversified immunoglobulin repertoire. Blood 104, 3647-3654.

Weller, S., Mamani-Matsuda, M., Picard, C., Cordier, C., Lecoeuche, D., Gauthier, F., Weill, J. C., and Reynaud, C. A. (2008). Somatic diversification in the absence of antigen-driven responses is the hallmark of the IgM+ IgD+ CD27+ B cell repertoire in infants. J. Exp. Med. 205, 1331-1342.

Whitlock, C. A., and Watson, J. D. (1979). B-cell differentiation in the CBA/N mouse. I. Slower maturation of mitogen and antigen-responsive $\mathrm{B}$ cells in mice expressing an X-linked defect. J. Exp. Med. 150, 1483-1497.

Wilkins, B. S., Davis, Z., Lucas, S. B., Delsol, G., and Jones, D. B. (2003). Splenic marginal zone atrophy and progressive CD8+ T-cell lymphocytosis in HIV infection: a study of adult post-mortem spleens from Cote d'Ivoire. Histopathology 42, 173-185.

Won, W. J., and Kearney, J. F. (2002). CD9 is a unique marker for marginal zone B cells, B1 cells, and plasma cells in mice. J. Immunol. 168, 5605-5611.

Wong, W. Y., Powars, D. R., Chan, L., Hiti, A., Johnson, C., and Overturf, G. (1992). Polysaccharide encapsulated bacterial infection in sickle cell anemia: a thirty year epidemiologic experience. Am. J. Hematol. 39, 176-182.

You, Y., Myers, R. C., Freeberg, L. Foote, J., Kearney, J. F., Justement, L. B., and Carter, R. H. (2011). Marginal zone B cells regulate antigen capture by marginal zone macrophages. J. Immunol. 186 , 2172-2181.

Zouali, M. (1995). B-cell superantigens: implications for selection of the human antibody repertoire. Immunol. Today 16, 399-405.

Zouali, M. (2007a). B cell superantigens subvert innate functions of $\mathrm{B}$ cells. Chem. Immunol. Allergy 93, 92-105.

Zouali, M. (2007b). Exploitation of host signaling pathways by B cell superantigens - potential strategies for developing targeted therapies in systemic autoimmunity. Ann. N. Y. Acad. Sci. 1095, 342-354.

Zouali, M. (2008). B lymphocytes chief players and therapeutic targets in autoimmune diseases. Front. Biosci. 13, 4852-4861.

Zouali, M. (2009). Natural antibodies. Encyclopedia of Life Sciences.
Available at: http://www.els.net (accessed September 2009).

Conflict of Interest Statement: The authors declare that the research was conducted in the absence of any commercial or financial relationships that could be construed as a potential conflict of interest.

Received: 24 August 2011; paper pending published: 14 September 2011; accepted: 04 November 2011; published online: 13 December 2011.

Citation: Zouali Mand Richard Y (2011)

Marginal zone B-cells, a gatekeeper of innate immunity. Front. Immun. 2:63. doi: 10.3389/fimmu.2011.00063

This article was submitted to Frontiers in Molecular Innate Immunity, a specialty of Frontiers in Immunology.

Copyright (c) 2011 Zouali and Richard. This is an open-access article subject to a non-exclusive license between the authors and Frontiers Media SA, which permits use, distribution and reproduction in other forums, provided the original authors and source are credited and other Frontiers conditions are complied with. 